\title{
A Wearable Tracking Device Inkjet-Printed on Textile
}

Bauyrzhan Krykpayev, Muhammad Fahad Farooqui, Rana Muhammad Bilal, Mohammad Vaseem and Atif Shamim

King Abdullah University of Science and Technology (KAUST), IMPACT Lab, Computer, Electrical and Mathematical Sciences and Engineering (CEMSE) Division, Thuwal 23955-6900, Saudi Arabia

Keywords: Tracking, E-textiles, Inkjet-Printing, Interface Layer method, Conductive Printing

\begin{abstract}
Despite the abundance of localization applications, the tracking devices have never been truly realized in E-textiles. Standard printed circuit board (PCB)-based devices are obtrusive and rigid and hence not suitable for textile based implementations. An attractive option would be direct printing of circuit layout on the textile itself, negating the use of rigid PCB materials. However, high surface roughness and porosity of textiles prevents efficient and reliable printing of electronics on textile. In this work, by printing an interface layer on the textile first, a complete localization circuit integrated with an antenna has been inkjet-printed on the textile for the first
\end{abstract}


time. Printed conductive traces were optimized in terms of conductivity and resolution by controlling the number of over-printed layers. The tracking device determines the wearer's position using WiFi and this information can be displayed on any internet-enabled device, such as smart phone. The device is compact $(55 \mathrm{~mm} \times 45 \mathrm{~mm})$ and lightweight $(22 \mathrm{~g}$ with $500 \mathrm{mAh}$ battery) for people to comfortably wear it and can be easily concealed in case discretion is required. The device operates at $2.4 \mathrm{GHz}$ communicated up to a distance of 55 meters, with localization accuracy of up to 8 meters.

\section{Introduction}

Wearable devices are making an impact on several applications ranging from medical, safety, leisure and entertainment. E-textiles or smart fabrics are usually contributing towards the wearable technology field [1-3]. E-textiles have been used to incorporate energy management [4], memory [5], sensors [6-7], electrochromic [8], energy harvesting [9], nano-generator [1012], battery [13], supercapacitors [14], communication [15-17] and health monitoring [18-22] based devices on or within fabrics to facilitate the human-device interaction. It is estimated by Grand View Research that global smart textile market will reach 1,500 million USD by 2020 [23].

Localization i.e. ability to track the position of an object is another research area which has seen a tremendous boom in past couple of years [24-26]. A diverse range of applications have benefited from advancements in this technology, ranging from health care monitoring; generic child, pet or vehicle tracking; to a number of complex location aware services such as context aware marketing, crowd management etc. Global navigation satellite system (GNSS) provides a very viable solution for outdoor tracking owing to its global availability and excellent 
localization accuracy. However, a globally available indoor or integrated (i.e. combined indoor and outdoor) tracking technology is a challenging problem, receiving major industrial and research interest.

Combining localization technology with wearable devices opens door for many exciting applications in areas such as health care, environmental sensing, child, pet, elderly monitoring etc. However, currently there are no true E-textiles tracking implementations. A few examples of clothes with tracking features are realized by gluing, stitching or pocketing regular trackers onto textile [27]. Such incorporation of trackers can hardly be labeled as E-textiles. Since these trackers are rigid PCB-based, so result is an uncomfortable and non-practical clothing. Moreover, since the incorporation of such PCB-based tracking devices on T-shirts or dresses is not feasible, such tracking devices are usually featured on heavy duty clothes i.e. protective uniforms, coats and ski jackets where the size and rigidness of trackers are of lesser concern [27]. True E-textiles implementation can potentially be accomplished via conductive printing since printing the circuit layout directly on textiles, can eliminate the rigidness coming from PCB substrate, as shown in our previous work [28]. This method is already widely exploited to print on different substrates including paper and plastic [29-32]. The advantage of conductive printing process lies in its similarity to PCB fabrication. Conductive printing allows producing entire electronic circuits in a planar, highly repeatable and low-cost manner. However, the main obstacle in conductive printing on textiles is the substrate itself. Common textiles are constructed from yarn that is twisted and interwoven. Due to the interlacing of warp and weft yarns, a textile structure is usually very rough and has significant dips that can be visibly seen. Such surface structure is highly unfavorable for direct conductive printing on textiles since ink will not be distributed evenly across the surface; thus, proper linking will not occur and reasonable 
conductivity will not be achieved. Consequently, to perform successful conductive printing, fabric surface should be made flat and uniform.

To overcome the roughnesses and porosity of textiles, a thick interface layer can be printed on the textile. The interface layer method can be an intermediate screen-printed polyurethane-based dielectric layer that can cover the surface profile of a material rendering it flat and relatively smooth. There are few reports utilizing the interface layer method, wherein simple structures consisting of interconnect, patches, and MEMS were studied [33-36]. However, a complete circuit or system on clothes for wearable electronic applications has never been demonstrated. Also, current research is bound only to a single dielectric paste material, Fabink UV-IF4 supplied by Smart Fabric Inks, Ltd (http://www.fabinks.com/). Unfortunately, this interface layer ink is expensive (340 USD/100 g). Other low cost dielectric materials have never been investigated for interface layer formation purposes. Optimizing a low cost general purpose dielectric material will ease the access to the interface layer method and decrease the overall cost of prototype production.

This work, for the first time, demonstrates a complete system printed on a polyester/cotton Tshirt, which enables tracking of the person who is wearing that T-shirt through a smart phone or any internet enabled device. A comparatively low cost (50 USD/100 g) dielectric material (Creative Materials 116-20 Dielectric ink) is used to print the interface layer through manual screen printing method. The circuit layout and antenna was inkjet printed on fabric with silver nano-particles based conductive ink and its conductivity on interface layer was optimized. Utilizing WiFi technology, this wearable tracking system can locate the position of lost children, senior citizens, patients or people in uniforms, lab coats, hospital gowns, etc. Investigation on the direct printing of circuit layouts on textiles can potentially lay down a strong foundation for 
future development of E-textiles and open the path for realization of diverse applications such as Internet of Things (IoT).

\section{Materials and methods}

2.1. Materials: UV-curable dielectric ink, $116-20$ (CM 116-20) is purchased from Creative materials (http://www.creativematerials.com/). The silver nanoparticle based ink, DGP 40LT15C, is purchased by Advanced Nano Products (http://anapro.com/) is used as the conductive ink. $100 \%$ cotton and 65\%/35\% polyester/cotton fabrics were purchased from general purpose clothing department stores. 85\%15\% polyester/cotton textile with the brand name "Luminex 310" was purchased from Klopman International (http://www.klopman.com/).

2.2. Printing of interface layer and conductive ink: The dielectric interface layer is printed by manual screen printing using PET stencil and Doctor Blade. The conductive printing is accomplished by ink-jet printing via special ink-jet printer Dimatix DMP 2831 printer. This printer utilizes one and ten picoliter cartridges to deposit conductive drops onto the substrate in a predefined pattern. The jetting process is all digital, thus is easily configurable. Ten picoliter cartridges are used to print the circuit layout. The distance between drops is critical to establish good structure conductivity. The drop spacing of $20 \mu \mathrm{m}$ was experimentally found to perform well providing sharp line edges and almost no side overspills. After printing, the deposited layers were each sintered for 20 mins at $130{ }^{\circ} \mathrm{C}$ in a thermal oven. The chosen curing process is suggested by ink manufacturer and also can be safely used with textile substrates selected for this work. The 20 min thermal sintering at $130{ }^{\circ} \mathrm{C}$ was performed after the printing of each two layers. 
2.3. Characterization of conductive film and dielectric interface layer: The structural properties were examined using scanning electron microscopy (FEI NovaNano FEG-SEM 630). The thickness and uniformity of printed features on substrates were performed using a surface profiler (Veeco Dektak 150) and 3D interferometry (Zygo, Newview 7300). The permittivity and loss tangent of the textile and interface layer stack up was measured using Agilent (E4991A) impedance analyzer.

2.4. Antenna design and its measurement: The inverted F-antenna (IFA) for the on-textile circuit was optimized in Ansys ${ }^{\mathrm{TM}}$ High Frequency Structure Simulator (HFSS). The simulated IFA model and corresponding parameters are given in supporting information Fig. S1 (b) and Fig.S1 (a) respectively along with the details of the antenna operation. This model and its simulation results are used to validate the antenna design printed on textile. The antenna is connected to a surface mount coaxial connector that is RF compatible with the nominal

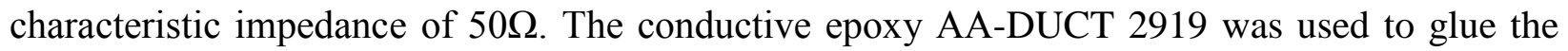
connector to the antenna feeding point.The fabricated antenna's reflection coefficients S11 were measured using Keysight N9923A FieldFox Vector Network Analyzer.

2.5. Localization prototyping on fabric: The prototype uses the ATMEGA328p in DIP package and also DIP socket whereas the final circuit will use the ATMEGA328p in TQFP package. Such change was administered to facilitate the testing of the on-textile prototype. The socket usage allowed for easy troubleshooting and debugging. Microcontroller houses the control code of tracking device, written in C++ using the Arduino development environment. Basically, the code operates the RN-171 WiFi module to scan ten nearby WiFi access points. This information is then sent to a server computer, by connecting to a known WiFi network, using the RN171 module. The control code is also responsible for establishing serial data communication 
between RN171 and the microcontroller, as well as operating a status LED. LED is needed mainly for indication and debugging purposes and can be eliminated if necessary.

\section{Results and discussions}

3.1. Printing on textiles: The interface layer method is suitable for production of smart fabrics from a verity of textile materials while preserving the natural elasticity of the clothes. To verify the versatility of this method, three common woven fabric substrates were selected - $100 \%$ cotton (black), 65\%/35\% polyester/cotton (white) and 85\%/15\% polyester/cotton (yellow). In this work, the manual screen printing, also called "Doctor Blading", is employed. The "Doctor Blading" technique is conceptually similar to the standard screen printing process, but, instead of an automatic or semi-automatic machine movement, the squeegee is slid manually. This method is not as accurate as the standard screen printing, but it is much more flexible and faster which is beneficial for prototype development. Furthermore, a screen of PET film with thickness of 0.3mm has been employed. PET film stencils can be easily and quickly altered using lasercutting or simple manual carving, thus allowing flexibility during screen printing. The process steps for interface layer formation are shown in Fig.1. The PET stencil is placed on top of the fabric textile (i), then CM116-20 dielectric paste is poured onto the stencil (ii). A squeegee is used to scrape dielectric paste across the stencil. Application force and angles should always be constant (iii). In order to achieve better surface roughness, the same procedure as in Step (iii) should be performed again but in the opposite direction (iv). Afterwards, the stencil is removed and the textile substrate is UV cured for $5 \mathrm{~min}$ at $1000 \mathrm{~mJ} / \mathrm{cm}^{2}(\mathrm{v})$. The textile substrate is placed in an oven for 1 hour at $130{ }^{0} \mathrm{C}$ (vi). During this step proper ventilation should be ensured. Notethat textiles in this research were tested to withstand such temperature without noticeable 


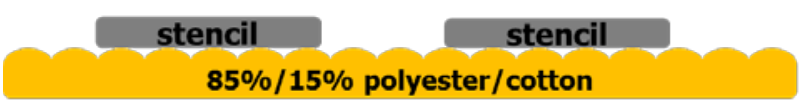

(i)

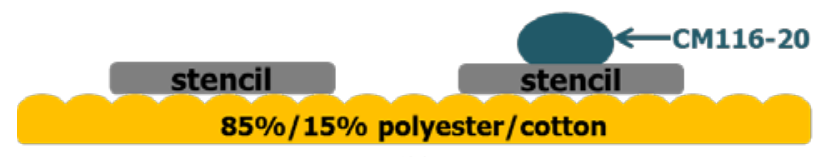

(ii)

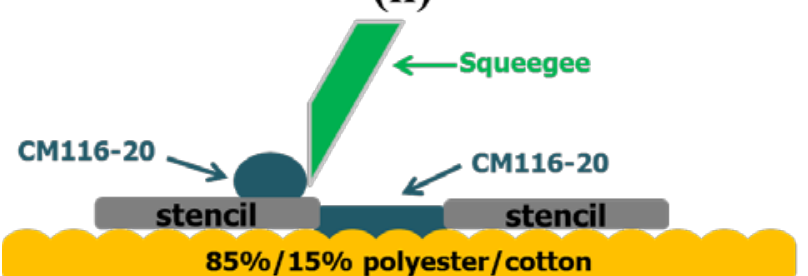

(iii)

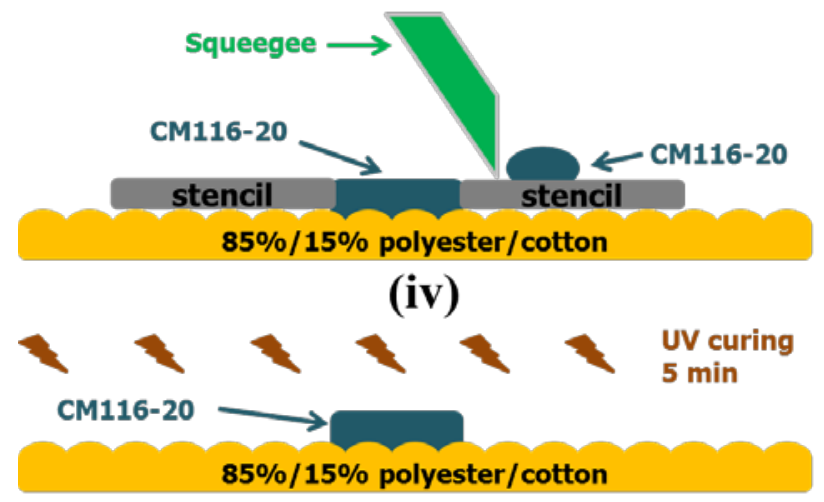

(v)

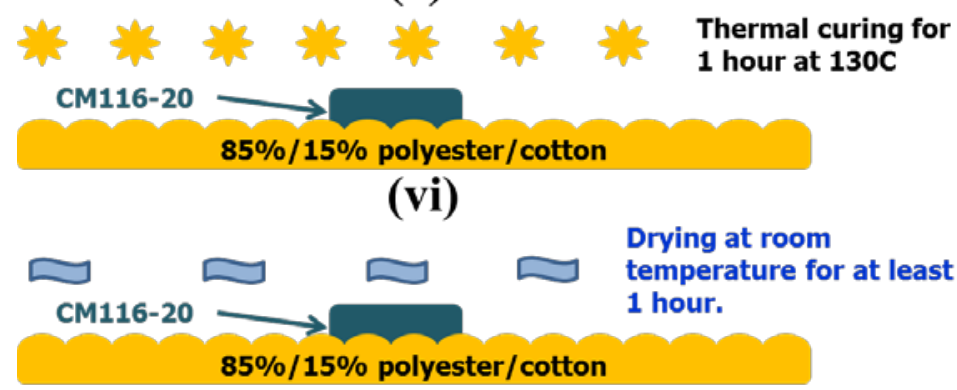

(vii)

Figure 1. Schematic diagram for interface layer deposition on textile.

degradation of textile qualities. However, textile flammability characteristics should be checked if any other textile material is considered as the substrate for the interface layer printing. Let the interface layer on textile dry at room temperature for at least 1 hour (vii). The resulting transparent yellowish tint interface layer on different textile materials formed by the general 


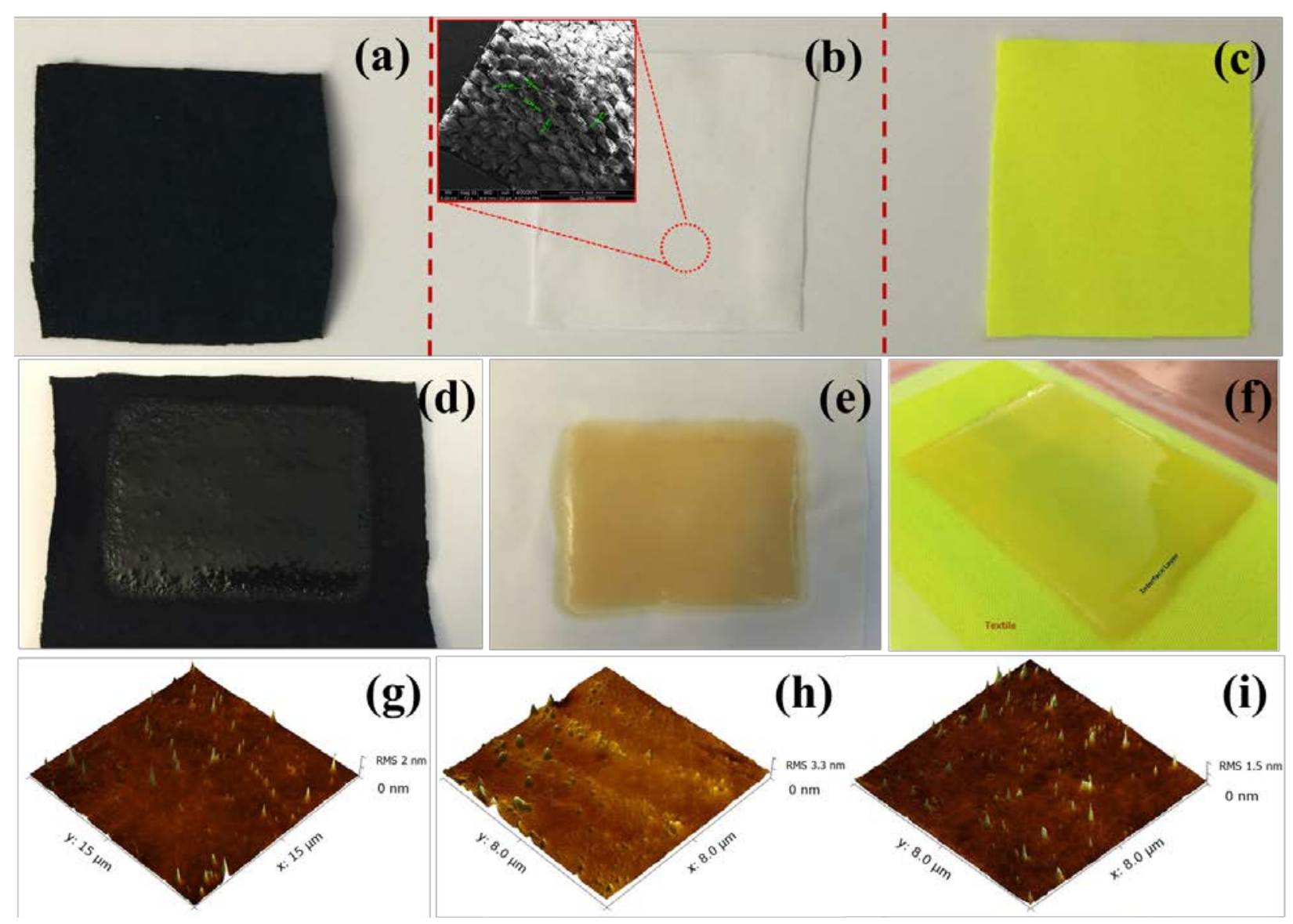

Figure 2. Bare textile substrate before printing (top), interface layers deposited (middle) and AFM surface roughness (bottom) on 100\% cotton (a, d \& g), 65\%/35\% polyester/cotton (b, e \& h) and $85 \% / 15 \%$ polyester/cotton (c, f \& i). The inset in (b) is showing surface SEM image of $65 \% / 35 \%$ polyester/cotton.

purpose CM116-20 dielectric paste is shown in Fig. 2. Usually fabrics are made of interlacing of warp and weft yarns (as shown in inset of Fig. 2b) which possess significant dips resulting in tens of micron of roughness. Printed interface layers are generally flat, uniform and clear. However, as can be seen in Fig. 2(d) the interface layer on $100 \%$ cotton is visibly coarse. Distinct hillocks can be seen all across the $100 \%$ cotton interface layer. These hillocks stem from the very high surface roughness of $100 \%$ cotton substrate. The stencil thickness is not enough to cover this roughness, thus some hillocks remain uncovered. Higher stencil thickness ( $>0.3 \mathrm{~mm})$ can be used to produce completely flat surface for $100 \%$ cotton substrate. Atomic Force 
Microscopy is used to measure the surface roughness of interface layers formed on $100 \%$ cotton 65\%/35\% polyester/cotton and 85\%/15\% polyester/cotton, as shown in Fig. 2 (g-i). There is an order of magnitude improvement and almost similar roughness is observed on these fabrics after interface layer. RMS surface roughness of these textile substrates are equal to $2 \mathrm{~nm}$ (g), $1.5 \mathrm{~nm}$ (h) and $3.3 \mathrm{~nm}$ (i), respectively. It is worth noting that AFM for 100\% cotton was made in the valleys between hillocks. These valleys possess flat surfaces.

\subsection{Morphological and electrical characterization of printed conductive features on}

interface/textiles: In order to study the electrical properties of conductive printing on the interface layer, 8 lines $(5 \times 1 \mathrm{~mm})$ and 8 rectangles $(10 \times 3 \mathrm{~mm})$ were printed with a number of overprinting layers using silver nanoparticles based ink (DGP 40LT-15C) on the interface layer formed on $100 \%$ cotton, $65 / 35 \%$ and $85 / 15 \%$ poly/cotton based textiles. The printed features were sintered at $130{ }^{0} \mathrm{C}$ for $20 \mathrm{~min}$. It is observed that initially with four printed layers, conductivity is low, i.e. $0.027-0.39 \times 10^{6} \mathrm{~S} / \mathrm{m}$. However, conductivity increases with the number of printed layers on these fabrics. Table 1 shows that the conductivity steadily improves; however, the highest conductivity of $1.43-2.08 \times 10^{6} \mathrm{~S} / \mathrm{m}$ is achieved with eight printed layers. It is obvious that with number of printed layers, density of conducting films increases and satisfied conductive pathways. The conductivity difference with six and eight printed layers is not too large, thus, it seems that six-conductive layers are optimal for the formation of printed structures. It should be noted that the conductivity of printed film is almost similar (in the range of $10^{6} \mathrm{~S} / \mathrm{m}$ ) on these textiles but the values are much lower than on traditional substrates i.e. PEN, PI. Usually, silver nanoparticles based inks require higher sintering temperature of more than $150{ }^{\circ} \mathrm{C}$ to achieve the conductivity in the range of $10^{7} \mathrm{~S} / \mathrm{m}$. But due to limited temperature constraint on these textiles, the temperature cannot go beyond $130{ }^{\circ} \mathrm{C}$. It might be a reason for lower 
conductivity on these textiles. But, there are several other sintering techniques available e.g., intense pulsed light, UV-curing, micro-wave, photonic, and laser curing which can lower the sintering temperature for highest conductivity, while remaining compatible with textiles. As the best conductivity of $1.85 \times 10^{6} \mathrm{~S} / \mathrm{m}$ with 6-layers is observed on the interface layer with $85 \% / 15 \%$ polyester/cotton, thus, this textile is chosen for final prototyping. After deciding optimal number of printed conductor layers and textile type for further experiments, the next step is to determine the minimum trace width that can be achieved by ink-jet printing. The minimum trace width is estimated using the simple arrangement shown in supporting information Fig. S2 (a). Two set of six lines, each were printed using three over-printing conductive layers with $10 \mathrm{~mm}$ lengths and different widths of $0.5,0.4,0.3,0.2,0.1$ and $0.05 \mathrm{~mm}$. The simple DC resistance measurement was performed by placing multimeter tips on line edges. Resulting DC resistance is represented in supporting information Fig. S2 (b). Traces up to $0.2 \mathrm{~mm}$ in width exhibited resistance of 1.41.5 $\Omega$. No DC resistance was measured for 0.1 and $0.05 \mathrm{~mm}$ traces, even though ink-jet printer can reliably print conductive lines up to 50 micron. Probable reasons can be high resistance for conductive traces due to very small width, excessive surface roughness or open circuits along the lines. For thinner line traces with conductive features, the printing and film morphology needs to be optimized further. Nonetheless, for printing the localization circuit, $0.2 \mathrm{~mm}$ conductor width is sufficient.

Table 1. Sheet-resistance, calculated resistivity and conductivity on different textiles.

\begin{tabular}{|c|c|c|c|c|c|c|c|c|c|}
\hline & \multicolumn{3}{|c|}{$100 \%$ cotton } & \multicolumn{3}{c|}{$65 / 35 \%$ poly/cotton } & \multicolumn{3}{c|}{$85 / 15 \%$ poly/cotton } \\
\hline Number of Layers & 4 & 6 & 8 & 4 & 6 & 8 & 4 & 6 & 8 \\
\hline Thickness $(\mu \mathrm{m})$ & 7 & 9 & 12 & 7 & 9 & 12 & 7 & 9 & 12 \\
\hline Sheet Resistance & 5332 & 610 & 58 & 262 & 70 & 47 & 370 & 60 & 40 \\
\hline
\end{tabular}




\begin{tabular}{|c|c|c|c|c|c|c|c|c|c|}
\hline$(\mathrm{m} \Omega / \square)$ & & & & & & & & & \\
\hline $\begin{array}{c}\text { Resistivity, } \rho(\mu \Omega . \mathrm{m}) \\
\begin{array}{c}\text { Conductivity, } \sigma\left(10^{6}\right. \\
\text { S/m) }\end{array}\end{array}$ & 37.3 & 5.49 & 0.7 & 1.83 & 0.63 & 0.56 & 2.59 & 0.54 & 0.42 \\
\hline
\end{tabular}

3.3. Bending test for printed conductive features on interface/textiles: The ability to remain conductive while being constantly subjected to bending is of paramount importance for Smart Textile. Ordinary clothes bend, curve and twist all the time. Interface layers formed on textiles will be exposed to repetitive bending. It is practical to measure whether conductive lines printed on interface layers can withstand several bending cycles. The fabrics with $100 \%$ cotton, 65\%/35\% and 85\%/15\% polyester/cotton are all bendable enough as it can be seen on Fig.3 (ac). However, to quantify the conductivity degradation, 6-layers of rectangles $(10 \times 5 \mathrm{~mm})$ were printed on interface layer formed on $85 \% / 15 \%$ polyester/cotton textile substrate. The bending test was performed under manual bending condition, i.e. the substrate was bent up to $~ 90$ degrees multiple times (50 times in total). After each 5 cycles of bending, the substrate was flattened and subsequently resistance was measured as shown in Supporting Table S1. We did not observe any significant resistance change with several bending cycles. Lines were also measured under bent conditions, and under extreme bending conditions, the resistance changes from 30 to $35 \%$ of its original value, however the resistance returns to its original value when the lines comes back to their original flat condition. 


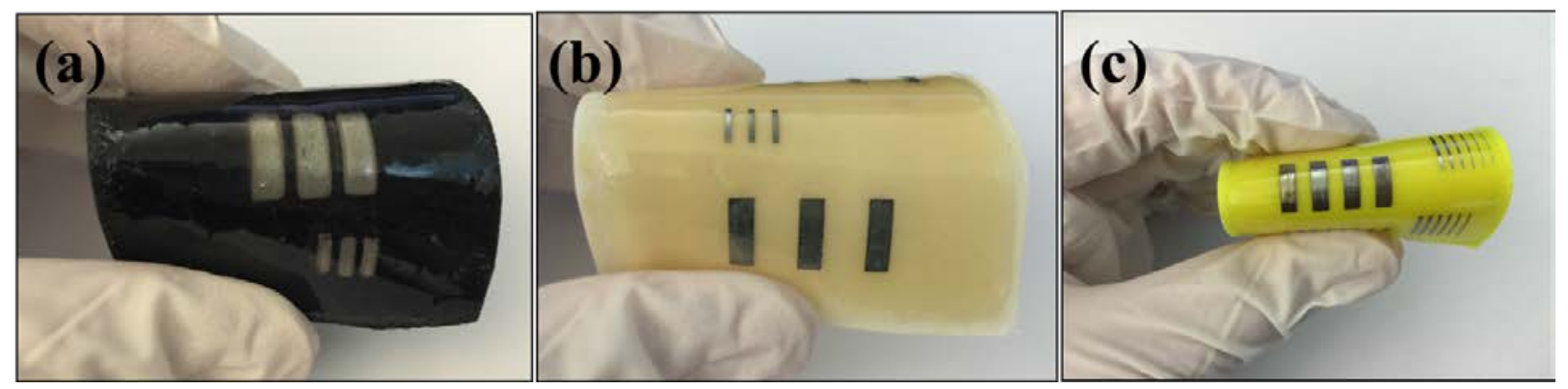

Figure 3. Interface layer bending with silver film supported on (a) $100 \%$ cotton, (b) 65\%/35\% polyester/cotton and (c) $85 \% / 15 \%$ polyester/cotton.

\subsection{System level design of the tracking device:}

The above experiments demonstrate the minimum feature sizes, conductivities and bending response achievable on textile fabric with dielectric interface layer method. This shows that it is possible to develop an E-textile system on a variety of textile fabrics using the proposed method.

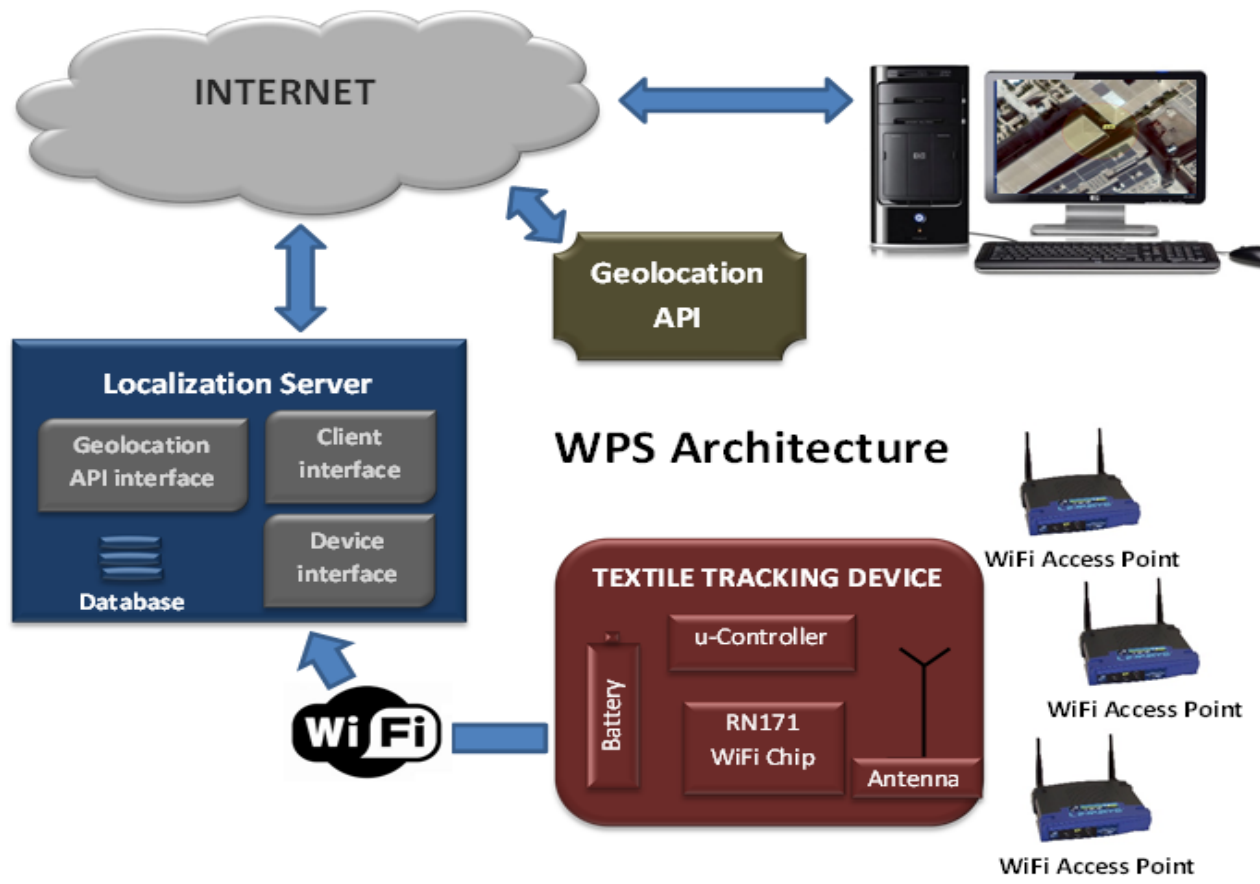

Figure 4. WiFi based tracking system. 
Before going into the details of fabrication of the proposed device, the system level design of the WiFi based tracking system is discussed here (Figure 4). The tracking device consists of a WiFi network processor, a microcontroller, battery and the WiFi antenna. The WiFi network processor provides the RF front-end in $2.4 \mathrm{GHz}$ band as well as the network stacks for the WiFi protocol. The microcontroller houses the control logic of the tracking device. The tracking device functions by scanning its environment for the WiFi signals. Each WiFi access point has a distinct MAC (Media Access Control) address. Tracking device collects MAC addresses and corresponding Received Signal Strength Indicators (RSSI) of the nearby access points. It then connects to a known WiFi network and sends this information to a server computer. The localization server receives this WiFi information from the textile tracking device, and processes it with the help of Google's geolocation API (Application Programming Interface). Google's geolocation API is a programming interface which enables estimation of location, by providing nearby access points (cellular and/or WiFi) information. It maintains an open source database, fed from millions of android and other devices around the globe, regarding information of cellular and WiFi access points. The API matches the provided WiFi information with its database and returns a location estimate. The localization server stores this location estimate in a local database on server computer, for further use. A JavaScript code on localization server, makes this location estimate available to the end user, by plotting it over a map in a browser window on any internet enabled device such as tablet, smart phone, PDA etc. The next section discusses realization of this tracking system, by first printing and optimizing WiFi antenna on textile and then direct printing of circuit layout on the textile. Afterwards, components integration on textile is done. 
3.5. Printed antenna on textile and its measured results: Electromagnetic properties of materials such as relative permittivity $\left(\varepsilon_{\mathrm{r}}\right)$ and loss tangent $(\tan \delta)$ are critical in the design of radio frequency (RF) structures such as antennas. Since the antenna is an integral part of any tracking device, the permittivity and loss tangent of interface layers on textile substrates should be acquired. As the textile substrate acts as a support for the printed interface layer, the separate analysis of the interface and textile layers is not possible. Therefore, the complete stack up of interface layer on textile material was considered for this analysis. The permittivity and loss tangent of the interface layer on different textiles is shown in Table 2. The values are quite close to each other for different cases. The small variation can be attributed to the different composition of the underlying textiles.

\section{Table 2. Properties of CM116-20 interface layer on different textiles}

\begin{tabular}{|l|l|l|}
\hline \multicolumn{1}{|c|}{ Textile Composition } & \multicolumn{1}{|c|}{$\boldsymbol{\varepsilon}_{\mathbf{r}}$} & \multicolumn{1}{|c|}{$\boldsymbol{}$} \\
\hline $85 \% / 15 \%$ polyester/cotton & 2.57 & 0.019 \\
\hline $65 \% / 35 \%$ polyester/cotton & 2.87 & 0.019 \\
\hline $100 \%$ cotton & 2.93 & 0.028 \\
\hline
\end{tabular}

In this work, a modified inverted F-antenna (IFA) is chosen for the proposed on-textile tracking device [37]. In a typical IFA, a shorting arm is used which acts as an inductor and nullifies the capacitive reactance of the antenna in order to have a pure real impedance. The length of this arm and hence the inductance is restricted by the gap between the IFA and the ground. In the modified design, the shorting arm of the IFA is bent around and brought close to the feed as shown in Fig. 5(a). The bending provides more length to the shorting arm and hence increases the inductance which is required to match the antenna to an impedance of 50 ohms. 
This technique reduces the required height of the IFA, thus making it even more low profiles. The antenna is designed to operate at $2.4 \mathrm{GHz}$. The IFA was printed, with the dimensions shown in Fig. S1 (a), on the interface layer on 85\%/15\% polyester/cotton textile. The antenna is realized by printing six conductive layers in three printing and thermal curing cycles at $130^{\circ} \mathrm{C}$ for 20 minutes. The resulting antenna metal thickness is $\sim 9 \mu \mathrm{m}$. The printed antenna on textile is shown in Fig. 5(a). The measurement of printed antenna is done in Satimo's Star Lab anechoic chamber. The measured return loss of the antenna is shown in Fig. 5(b) along with the simulated results. From the graphs, it is clear that the antenna is well matched at $2.4 \mathrm{GHz}$. The fabricated antenna has return loss of $20 \mathrm{~dB}$ at $2.4 \mathrm{GHz}$ and bandwidth of nearly $300 \mathrm{MHz}$. It can be seen that the measured results are close to the simulation. When the fabricated antenna is placed on a human body, the proximity effects can affect the performance of the antenna. However, as seen in Fig.

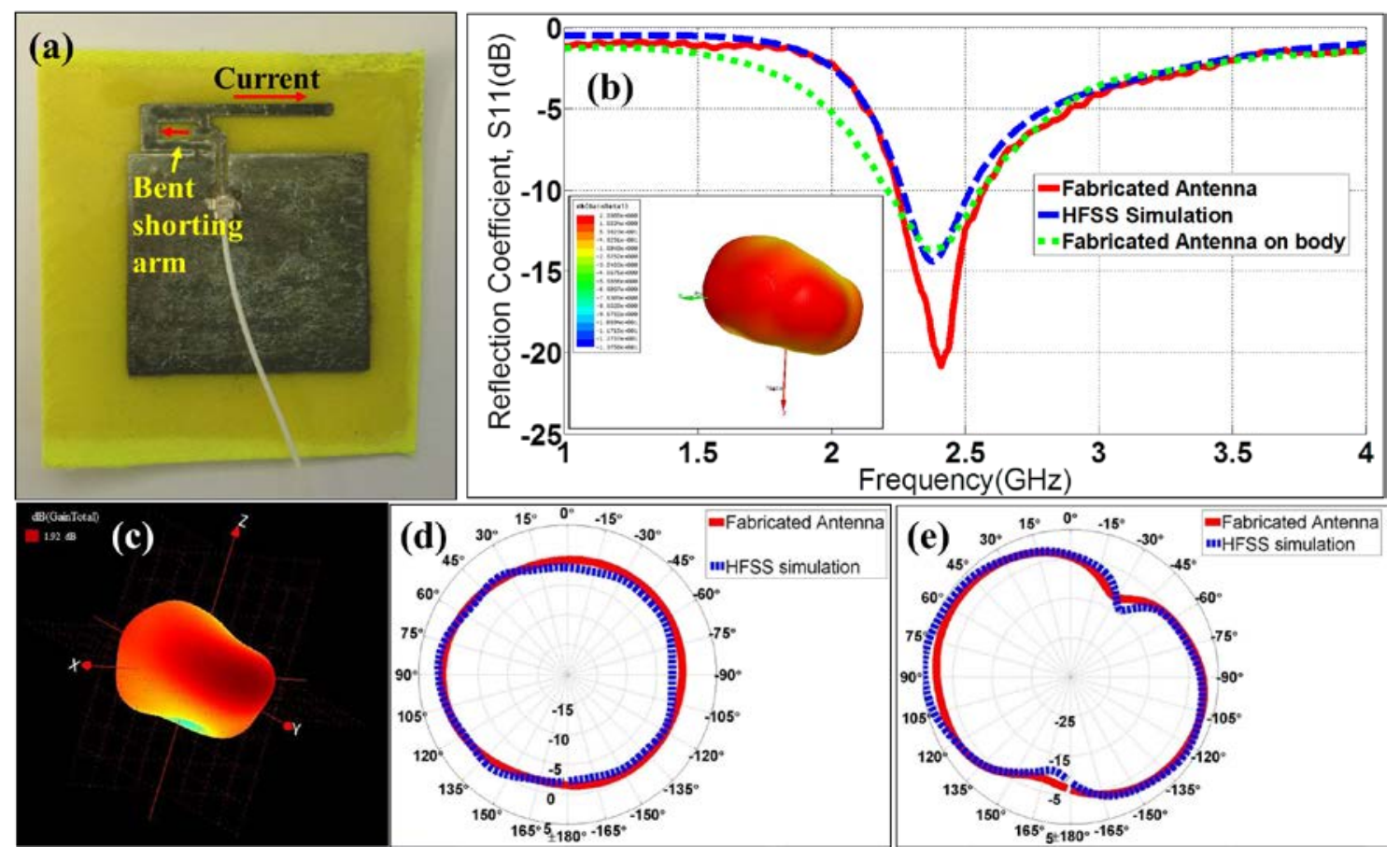

Figure 5. Antenna characterization. (a) Printed antenna on textile (b) Return loss (c) Measured radiation pattern (d) H-plane measurements (f) E-plane measurements. The inset in (b) is showing simulated radiation pattern in HFSS. 
5(b), the antenna performance does not change much. This is probably due to the fact that the antenna is not directly attached to the body and there is an air gap between the body and the textile on which the antenna is realized. However, if the clothing is skin-fit, the performance will be affected and modifications will need to be done in the antenna design in order to minimize the body effects. Measured 3D radiation patterns of the antenna are presented in Fig. 5 (c). Both simulated (inset of Fig. 5 (b)) and measured patterns show that the antenna is omni-directional. The omni-directional pattern is important for this application as the antenna needs to radiate equally along the horizon in order to establish communication with the WiFi access points. The simulated gain is $2.58 \mathrm{dBi}$ whereas the measured gain is $1.92 \mathrm{dBi}$. The difference of $0.56 \mathrm{~dB}$ can stem from different factors such as fabrication tolerance, and connector loss that were not accounted for in the simulation. The 2D radiation patterns in H-plane and E-plane are shown in Fig. 5(d) and 5(e) respectively. The radiation patterns are very similar to the standard IFA with a slight difference in the E-plane which is tilted. This tilt can be attributed to the current flowing in the shorting arm of the IFA. Due to the bending of the shorting arm, the current flow is in the opposite direction to the main radiating arm, Fig. 5(a), and hence can disturb the radiation from the antenna.

3.6. Printed circuit layout and components integration on textile: The next step after optimization of the antenna was to select IC components, develop schematic and circuit layout for the desired system. Roving networks, RN171 WiFi module was chosen as it requires very few external components and has an onboard TCP-IP stack for network processing. Atmel's ATMEGA328 controller was used to house the control logic of tracking device. The developed schematic and the circuit layout (designed in EAGLE CAD) are given in supporting information Fig. S3. This circuit layout was then realized on textile, using the interface layer method and the 
inkjet printing of conductive silver ink on the interface layer, as shown in Fig. 6(a). Conductive printing is accomplished via three printing and sintering cycles at $130{ }^{\circ} \mathrm{C}$ for 20 mins to produce total of six silver layers. The traditional soldering cannot be employed to bond the components to the printed circuit layout. Instead, the conductive epoxy is used as shown in Fig.6 (b). AADUCT 2919 conductive epoxy from Atom Adhesives is used because of its elasticity properties that are useful in flexible circuits such as circuits on textile. However, the conductive epoxy
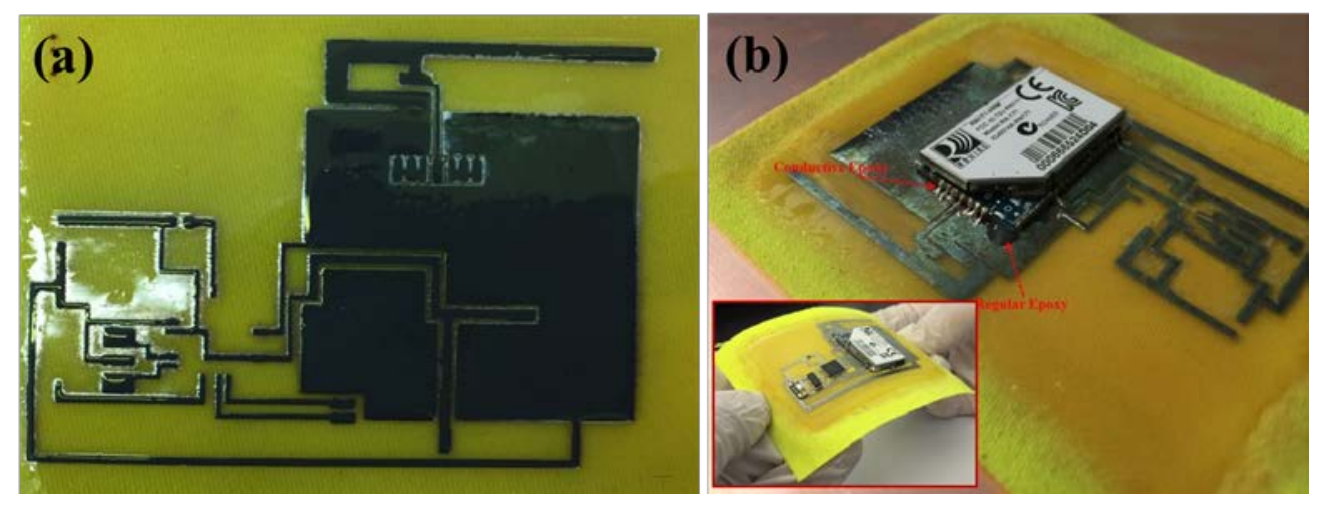

Figure 6. (a) Circuit layout printed on the interface layer (b) Components integration. The inset in (b) is flexibility of the device on textile.

alone is not enough to establish strong bonding between discrete components such as RN171 WiFi module and the interface layer. Also, without additional attachment, the conductive epoxy is easily broken when the textile substrate is bent. A regular epoxy is used for additional strength to the components attached to the substrate as shown in Fig. 6 (b). For this work 5-minute epoxy from Devcon is applied but any epoxy can be used as well. Once all components are attached to the printed circuit board, the on-textile prototype is assembled. As can be seen in inset of Fig. 6 (b), the final localization device is still flexible as compare to a rigid PCB, however it has lost some flexibility due to mounting of the components. In future, flexible un-packaged chips 
(integrated circuit dies) [38] can be used to further enhance the flexibility of the wearable system. A rechargeable $3.7 \mathrm{~V}, 5 \times 30 \times 35 \mathrm{~mm}$, flexible lithium polymer (LiPo) battery is used to power the tracking device, in this work. The battery's capacity is $500 \mathrm{mAh}$ and it can last up to 33 hours with one transmit and receive function per minute. LiPo batteries are available in custom shapes and sizes, and can be easily chosen to match the requirements of any specific application. Also, depending on the number of transmit and receive signals the battery lifetime can be dramatically changed. Thus the battery shown might be replaced with a smaller battery, whilst maintaining the same battery life by reducing location update frequency of the device.

3.7. Field tests of on-textile localization prototype: The operation of the on-textile localization prototype developed in the previous section needs to be tested both in free space (air) and on a human body, for stable performance in actual conditions. It is difficult to simulate accurately the influence of a human body on the localization performance, therefore the field tests are needed to showcase that the localization circuit remains fully functional while being worn. Device radiation pattern measurements fall under active antenna testing that is used to confirm that the antenna is functional when incorporated into the final circuit and fed from the microcontroller. During the test, the E-plane and H-plane of the antenna are measured. Since it is an active testing, the real world conditions should be simulated, thus the measurements were not carried out in an anechoic chamber. Instead, manual measurement setup, shown in supporting information Fig. S4 (a) is employed. It is worth noting that the manual radiation pattern measurement provides an approximate estimation of antenna radiation patterns. The measurement is carried out using transceiver and receiver. The tracking device, Device Under Test (DUT), is programmed as a transceiver, and RN171 evaluation board is configured as a receiver and connected to the laptop. The combination of the receiver and a laptop is capable of 
reading the signal transmitted from the DUT. The DUT provides $12 \mathrm{dBm}(16 \mathrm{~mW})$ of maximum transmitted RF power, whereas receiver's sensitivity is $-83 \mathrm{dBm}$. The device is kept stationary while the receiver is rotated around the DUT in $15^{\circ}$ steps. At each point the RSSI measurement was recorded. The radius of the rotation circle should be in the far-field and should always be constant. For this test, the radius of $75 \mathrm{~cm}$ was selected. The measured E-plane and H-plane in air are represented in supporting information Fig. S4 (b) \& (c). The H-plane can be considered omnidirectional with small variation of $7 \mathrm{~dB}$, mainly in the $90^{\circ}$ direction. The embedded circuitry that is located in the same direction may be the reason behind the variation. The E-plane is very similar to the E-field measured during the anechoic characterization of the standalone antenna.

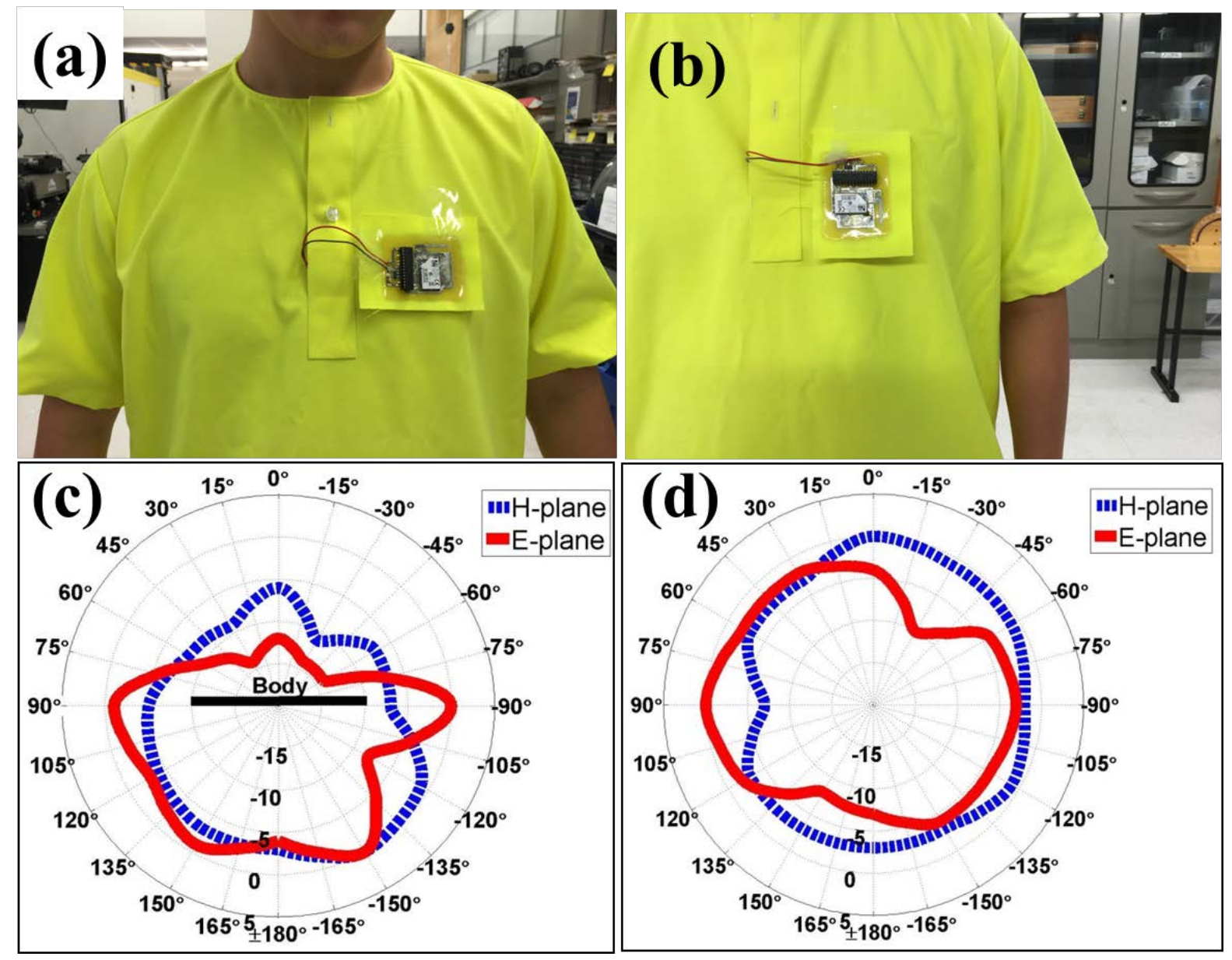


Figure 7. Radiation pattern measurements on body (a) E-plane (b) H-plane, (c) curves in E \& $\mathrm{H}$-plane and (d) on free space

For on-body testing, the DUT is attached to the T-shirt worn by a consenting human. The rest of the experiment is carried out in the same fashion to the in air measurements. The evaluated Eplane and H-plane on the body are represented on Fig. 7. It can be seen that both $\mathrm{E}$ and $\mathrm{H}$ planes are heavily affected in the direction of human body location $\left(90^{\circ}\right.$ to $0^{\circ}$ to $-90^{\circ}$ region) while the opposite direction $\left(90^{\circ}\right.$ to $180^{\circ}$ to $\left.-90^{\circ}\right)$ exhibits the variation in the radiation pattern of $-7 \mathrm{~dB}$. Such phenomenon can be explained by the absorption of radiation by a human body. The human body consists mainly of water and other organic tissues that possess very high dielectric constants and loss tangents. Thus, the human body tends to absorb the radiation that clearly can be seen on Fig. 7 (c) where the radiation in the wearer's direction is significantly attenuated. As it was shown before, the effect of the human body on the device radiation pattern is investigated using active testing. Since the degradation of the radiation field in the body direction was expected, and the radiation pattern variation in the out of the body direction is adequate and resembles free space radiation, the antenna can be used for E-textiles in wearable applications.

\subsection{Communication distance and accuracy for printed tracking devices on fabric: To} quantify the transmitting and receiving range of the device under discussion, communication distance measurements were made. Similar to the device radiation pattern measurements, the ontextile prototype was programmed as a transmitter with $12 \mathrm{dBm}$ TX power. The RN-171 evaluation board was configured as a receiver with $\mathrm{RX}$ sensitivity of $-83 \mathrm{dBm}$. The test was administered by placing both the transceiver and receiver on position zero and then moving the transceiver away from the receiver in five-meter steps. At each point the RSSI measurement was recorded. The test was carried out in an open area on the university campus to reproduce actual operational circumstances. The measurements have been taken both in air and on-body by 
attaching the device on the T-shirt worn by a consenting human (Fig. 8 a). The measurement results shows that in the air the communication distance of up to 70 meters can be achieved. The distance is reduced to a maximum of 55 meters when the device is tested on the human body. Both of these values are adequate for the targeted applications. It can be seen that with increasing distance the measured RSSI values tend to cluster in -70 to $-80 \mathrm{dBm}$ range that is very close to the receiver sensitivity limit. Thus, improving the receiver sensitivity (for ex. to $-100 \mathrm{dBm}$ ) can easily increase the potential communication distance for both in the air and on-body setups.

(a)

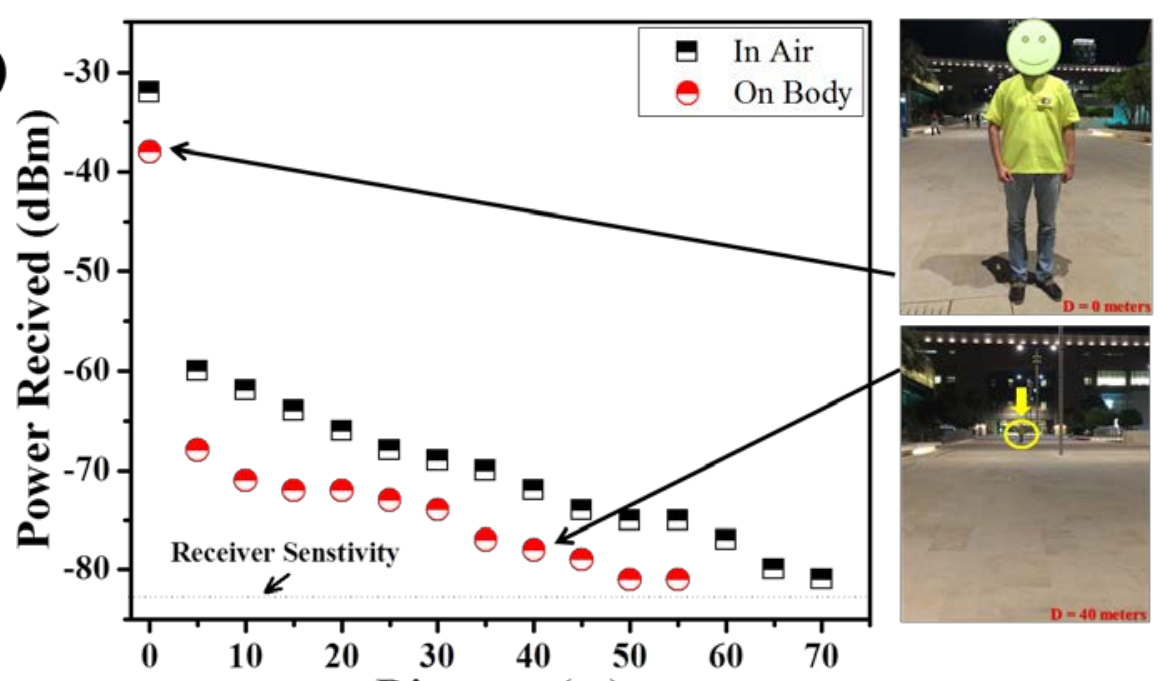

(b)

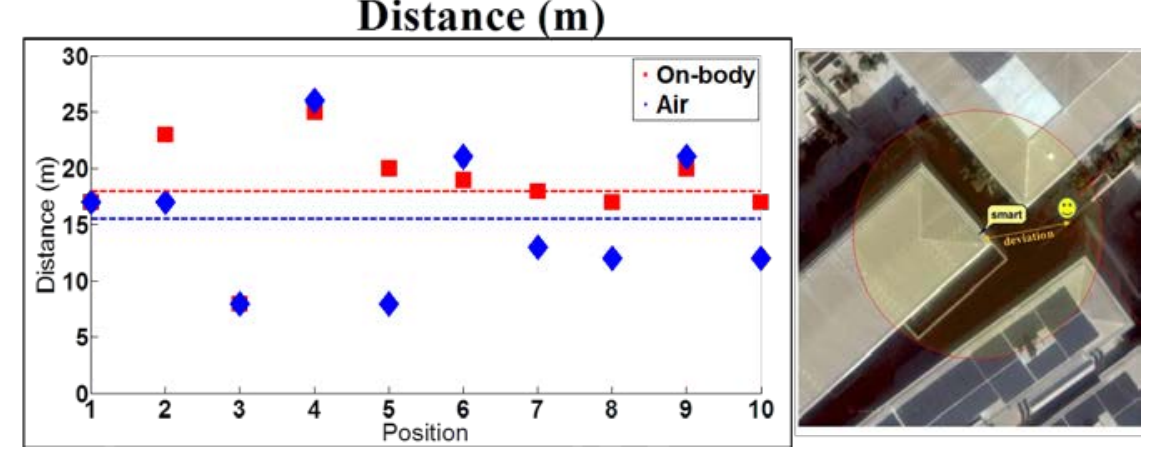

Figure 8. (a) Communication distance measurements on-body at 0 meters and 40 meters; (b) transmitting distance results for on body and in air with accuracy measurement setup.

To evaluate the positioning accuracy of the textile tracking device, ten different locations across KAUST campus were selected. Their latitudes and longitudes were recorded. Human test subject 
wearing T-shirt with the attached localization device prototype was placed exactly on each of these locations i.e. actual location [as represented by smile face shown in Fig. 8 (b)]. The estimated location was determined via the localization prototype both in air and on body. Estimated latitudes, longitudes and accuracies were recorded, and the accuracy circles were plotted on the map. The resulting distance deviation (accuracy) for in-air and on-body varies from 8 to 26 meters. Fig. 8 (b) shows that the average for in-air and on-body deviation is 15.5 and 18 meters respectively. Of course, the derived localization accuracy of 8-26 meters is not an ideal tracking accuracy. However, the WiFi localization technology is constantly improving as more access points are being entered in the database and new triangulation algorithms are being developed. Nevertheless, the localization accuracy of 8-26 meters is more than enough for a vast number of different applications. Moreover, due to the better filling of Google database in more populous areas, the tracking accuracy of the proposed WiFi localization system can potentially be much better in such regions.

\section{Conclusions}

In conclusion, we present, for the first time, the inkjet-printing of a complete localization circuit and antenna on textile via the Interface Layer method. The Interface Layer method is added benefit for its manufacturing easiness and preserves the textile flexibility. The conductive features are optimized on interface layer in terms of printed resolution and conductivity with number of over-printing layers. Since the antenna is an integral part of any tracking device, inverted F-antenna (IFA) is printed on textile and measured. The on-textile tracking device was produced via optimized conductive printing on the CM116-20 interface layer followed by the electronic components attachment. The resulting device was field tested and showed the communication distance of up to 55 meters and localization accuracy of up to 8 meters while 
being worn on a body. The developed localization device is flexible and small enough for people to comfortably wear it on clothes such as uniforms, lab coats, nursing gowns, etc. This device is connected to the server, thus its position can be determined from any internet-enabled device such as a mobile phone or a laptop.

\section{Supporting information}

Table consisting bending assessment for silver film based on measured DC resistance on 85\%/15\% polyester/cotton, Printed fine line features with different width on fabric and its corresponding measured resistance, Table showing parameters for inverted printed antenna and localization circuit's antenna design and dimensions in HFSS, The schematic localization circuit design, Device radiation pattern measurements setup and radiation pattern measurements in free space.

\section{Acknowledgments}

We acknowledge financial support from King Abdullah University of Science and Technology (KAUST) Office of Sponsored Research (OSR).

\section{Refrences}

[1] Rossi D D 2007 Electronic textiles: A logical step Nat. Mater. 6 328-329.

[2] Matsuhisa N, Kaltenbrunner M, Yokota T, Jinno H, Kuribara K, Sekitani T, Someya T 2015 Printable elastic conductors with a high conductivity for electronic textile applications Nat. Commun. 6 7461-7471. 
[3] Yetisen A K, Qu H, Manbachi A, Butt H, Dokmeci M R, Hinestroza J P, Skorobogatiy M, Khademhosseini A, Yun S H 2016 Nanotechnology in Textiles ACS Nano 10 3042-3068.

[4] Guo Y, Li K, Hou C, Li Y, Zhang Q, Wang H 2016 Fluoroalkylsilane-Modified TextileBased Personal Energy Management Device for Multifunctional Wearable Applications ACS Appl. Mater. Interfaces 8 4676-4683.

[5] Son D, Koo J H, Song J K, Kim J, Lee M, Shim H J, Park M, Lee M, Kim J H, Kim D-H 2015 Stretchable Carbon Nanotube Charge-Trap Floating-Gate Memory and Logic Devices for Wearable Electronics ACS Nano 9 5585-5593.

[6] Ha M, Park J, Lee Y, Ko H 2015 Triboelectric Generators and Sensors for Self-Powered Wearable Electronics 2015 ACS Nano 9 3421-3427.

[7] Lee J, Kwon H, Seo J, Shin S, Koo J H, Pang C, Son S, Kim J H, Jang Y H, Kim D E, Lee T 2015 Conductive Fiber-Based Ultrasensitive Textile Pressure Sensor for Wearable Electronics Adv. Mater. 27 2433-2439.

[8] Yan C, Kang W, Wang J, Cui M, Wang X, Foo C Y, Chee K J, Lee P S 2014 Stretchable and Wearable Electrochromic Devices ACS Nano 8 316-322.

[9] Li S, Zhong Q, Zhong J, Cheng X, Wang B, Hu B, Zhou J 2015 Cloth-Based Power Shirt for Wearable Energy Harvesting and Clothes Ornamentation ACS Appl. Mater. Interfaces 7 14912-14916.

[10] Seung W, Gupta M K, Lee K Y, Shin K-S, Lee J-H, Kim T Y, Kim S, Lin J, Kim J H, Kim S-W 2015 Nanopatterned Textile-Based Wearable Triboelectric Nanogenerator ACS Nano 9 3501-3509.

[11] Zhong J, Zhang Y, Zhong Q, Hu Q, Hu B, Wang Z L, Zhou J 2014 Fiber-Based Generator for Wearable Electronics and Mobile Medication ACS Nano 8 6273-6280.

[12] Zhou T, Zhang C, Han C B, Fan F R, Tang W, Wang Z L 2014 Woven Structured Triboelectric Nanogenerator for Wearable Devices ACS Appl. Mater. Interfaces 6 1469514701.

[13] Lee Y-H, Kim J-S, Noh J, Lee I, Kim H J, Choi S, Seo J, Jeon S, Kim T-S, Lee, J-Y, Choi J W 2013 Wearable Textile Battery Rechargeable by Solar Energy Nano Lett. 13 5753-5761.

[14] Zhang D, Miao M, Niu H, Wei Z 2014 Core-Spun Carbon Nanotube Yarn Supercapacitors for Wearable Electronic Textiles ACS Nano 8 4571-4579. 
[15] Chauraya A, Whittow W, Vardaxoglou J, Li Y, Torah R, Yang K, Beeby S, Tudor J 2013 Inkjet printed dipole antennas on textiles for wearable communications Microwaves, Antennas Propagation, IET 7 760-767.

[16] Hussain A M, Ghaffar F A, Park S I, Rogers J A, Shamim A, Hussain M M 2015 Metal/Polymer Based Stretchable Antenna for Constant Frequency Far-Field Communication in Wearable Electronics Adv. Func. Mater. 25 6565-6575.

[17] Huang X, Leng T, Zhu M, Zhang X, Chen J C, Chang K, Aqeeli M, Geim A K, Novoselov K S, Hu Z 2015 Highly Flexible and Conductive Printed Graphene for Wireless Wearable Communications Applications Sci. Rep. 5 18298-18306.

[18] Kang T-H, Merritt C, Grant E, Pourdeyhimi B, Nagle H 2008 Nonwoven fabric active electrodes for biopotential measurement during normal daily activity Biomedical Engineering, IEEE Transactions on 55 188-195.

[19] Yoo J, Yan L, Lee S, Kim H, Yoo H-J 2009 A wearable ecg acquisition system with compact planar-fashionable circuit board-based shirt Information Technology in Biomedicine, IEEE Transactions on 13 897-902.

[20] Yoo J, Yan L, Lee S, Kim Y, Yoo H-J 2010 A 5.2 mw self-configured wearable body sensor network controller and a $12 \mathrm{mu}$ w wirelessly powered sensor for a continuous health monitoring system Solid-State Circuits, IEEE Journal of 45 178-188.

[21] Paul G, Cao F, Torah R, Yang K, Beeby S, Tudor J 2014 A smart textile based facial EMG and EOG computer interface. Sensors Journal, IEEE 14 393-400.

[22] Takei K, Honda W, Harada S, Arie T, Akita S 2015 Toward Flexible and Wearable HumanInteractive Health-Monitoring Devices Adv. Healthcare Mater. 4 487-500.

[23] James S 2015 Smart textile fabric market to reach USD 1.59 billion by 2020: Grand view research, inc. http:/www.prnewswire.com/news-releases/smart-textilefabric-market-toreach-159-billion-by-2020-grand-view-research-inc-521228641.html/

[24] Thimmesch D 2015 3D Printed GPS Tracker Improves Quality of Life for People with Alzheimer’s. May 5 https://3dprint.com/62214/gps-tracker-for-alzheimers/

[25] Jais M I, Jamlos M F, Jusoh M, Sabapathy T, Kamarudin M R 2013 A Novel 1.575-GHz Dual-Polarization Textile Antenna for GPS Application Microwave and Optical Technology Letters 55 2414-2420. 
[26] Salman N, Alsindi N, Mihaylova L, Kemp A 2014 Super resolution wifi indoor localization and tracking in Sensor Data Fusion: Trends, Solutions, Applications (SDF) 1-5.

[27] Technovelgy, "Navjacket guides skiers with built-in display, headphones," accessed: 201510-29. http://www.technovelgy.com/ct/Science-Fiction-News.asp?NewsNum=1354.

[28] Krykpayev B, Farooqui M F, Bilal R M, Shamim A 2016 A WiFi Tracking Device Printed Directly on Textile for Wearable Electronics Applications IEEE MTT-S International Microwave Symposium (IMS) 1-4.

[29] Rida A, Yang L, Vyas R, Tentzeris M 2009 Conductive inkjet-printed antennas on flexible low-cost paper-based substrates for rfid and wsn applications Antennas and Propagation Magazine, IEEE 51 13-23.

[30] McKerricher G, Gonzalez J, Shamim A 2015 Fully Inkjet Printed RF Inductors and Capacitors using Polymer Dielectric and Silver Conductive Ink with Through Vias IEEE Transactions on Electron Devices (TED) 62 1002-1009.

[31] Öhlund T, Schuppert A K, Hummelgård M, Bäckström J, Nilsson H-E, Olin H 2015 Inkjet Fabrication of Copper Patterns for Flexible Electronics: Using Paper with Active Precoatings ACS Appl. Mater. Interfaces 7 18273-18282.

[32] Vaseem M, McKerricher G, Shamim A 2016 Robust Design of a Particle-Free SilverOrgano-Complex Ink with High Conductivity and Inkjet Stability for Flexible Electronics ACS Appl. Mater. Interfaces 8 177-186.

[33] Whittow W, Chauraya A, Vardaxoglou J, Li Y, Torah R, Yang K, Beeby S, Tudor J 2014 Inkjet-printed microstrip patch antennas realized on textile for wearable applications Antennas and Wireless Propagation Letters, IEEE 13 71-74.

[34] Whittow W, Li Y, Torah R, Yang K, Beeby S, Tudor J 2014 Printed frequency selective surfaces on textiles Electronics Lett. 50 916-917.

[35] Li Y, Torah R, Beeby S, Tudor J 2015 Fully direct-write dispenser printed dipole antenna on woven polyester cotton fabric for wearable electronics applications Electronics Letters 51 1306-1308.

[36] Kiourti A, Lee C, Volakis J 2015 Fabrication of textile antennas and circuits with $0.1 \mathrm{~mm}$ precision Antennas and Wireless Propagation Letters, IEEE PP 1-1.

[37] Audun A 2008 2.4GHz Inverted F Antenna. Design Note DN00007, Texas Instruments 
[38] Hackler D, Sime D G, Wald S F 2015 Enabling electronics with physically flexible ICs and hybrid manufacturing. Proc. IEEE 103 633-643. 